\title{
Causes, Mechanisms of Creation and Clinical Peculiarity of Hypoglycemia in Infants, Children and Adolescents
}

\author{
Vyacheslav Semionovich Lukyanchikov, Alim Nasimovich Scharapov \\ Research Institute of Age Physiology of the Ministry of Education of the Russian Federation, Moscow, Russia \\ Email address: \\ vsluk@yandex.ru (V. S. Lukyanchikov)

\section{To cite this article:} \\ Vyacheslav Semionovich Lukyanchikov, Alim Nasimovich Scharapov. Causes, Mechanisms of Creation and Clinical Peculiarity of \\ Hypoglycemia in Infants, Children and Adolescents. Clinical Medicine Research. Vol. 10, No. 2, 2021, pp. 40-46. \\ doi: $10.11648 /$ j.cmr.20211002.12
}

Received: January 15, 2021; Accepted: January 23, 2021; Published: April 7, 2021

\begin{abstract}
Low blood glucose is a dangerous violation of homeostasis, especially for infants, children and adolescents, in whom hypoglycemia is often observed, which is explained by immaturity and insufficient efficiency of adaptation reactions, primarily imperfection of hormonal regulators and instability of enzyme systems of glucose metabolism and alternative energy sources, in the form of free fatty acids, ketones, lactate, etc. The special danger of hypoglycemia in children is that glucose, although not single, but a priority source of energy for the nervous structures, therefore the lack of glucose leads to a delay in intellectual development and even to brain damage. At the same time, the lack of stability and clarity of reactions from the central nervous system, somatic and autonomic divisions of the nervous systems characteristic of these age groups, makes it extremely difficult to recognize the hypoglycemic syndrome (HS), and the etiological and pathogenetic diversity of the HS makes its clarifying diagnosis an extremely difficult task. In this regard, the issues of prevention, timely diagnosis, urgent and subsequent therapy of hypoglycemia in newborns, children and adolescents are very relevant. Fulfilled critical analysis of thematic literature over many years, as well as analysis own clinical and research experience. The article discusses the typology of HS from the standpoint of the causes and mechanisms of development, establishes anamnestic and obstetric-gynecological features of the course of pregnancy and childbirth, which predispose to hypoglycemia, clarifies the anthropological and clinical stigmas of HS in the fetus and newborn, which suggests the possibility of hypoglycemia in a child, shows the features clinical manifestations of HS in children of different ages. For clinical practice, an etiological and pathogenetic classification of HS, an algorithm for the treatment of hypoglycemic coma (HC), a scheme for the pathogenetic diagnosis of HS is proposed. Hypoglycemia in infants, children and adolescents is a common syndrome that poses a great danger to the health and development of the child and requires timely and qualified assistance.
\end{abstract}

Keywords: Hypoglycemia, Hypoglycemic Coma, Emergency Endocrinology

\section{Introduction}

One of the largest sections of human pathology is made up of diseases and syndromes of adaptation, or rather misadaptation. We are talking about an excessive or insufficient response of the immune, nervous and endocrine systems to endogenous and exogenous factors, to which the body must adapt or neutralize. Misadaptation can form at the level of organs and individual systems, which inadequately respond to the normal adaptive signals of systemic regulators [1]. The constant sphere of realization of adaptive reactions is the internal medium of the body - homeostasis, especially energy metabolism, and above all the production, utilization and level of glucose in the blood, which, normally, within the framework of adaptation reactions, varies widely from 3 to 10 $\mathrm{mmol} / \mathrm{L}$.

A common form of inadequate metabolic adaptation is hypoglycemia - a decrease in blood glucose levels below the physiological minimum. This is the so-called functional hypoglycemia, along with which there are many other etiological and pathogenetic variants of hypoglycemic syndrome (HS). Many of these options are relevant, sometimes selectively relevant specifically for infants, children and adolescents [2-5].

In general, the analysis of thematic literature, case studies and clinical experience ought to show the role of 
hypoglycemia as an essential factor in the pathogenesis of adaptation diseases and as a marker of adaptive responses.

\section{Methods}

When preparing the article, a comparative analysis of thematic publications related to the etiology, pathogenesis and clinical features of HS in children at different age periods was carried out. The analysis of own clinical observations of HS in children in multidisciplinary clinics in Moscow and region is carried out. The results of scientific research carried out in the laboratory of complex studies of the problems of adaptation of children and adolescents of the Research Institute of Age Physiology of the Ministry of Education of the Russian Federation are used. On this basis, some aspects of HS have been clarified and updated, namely: 1. Etiology and pathogenesis of the most frequent forms of HS in infants, children and adolescents. 2. Clinical features of HS in these age groups. 3. Practical approaches to the prevention, diagnosis and treatment of HS in these age groups. The results are presented below.

\section{Results}

The definitions of HS and HC were formulated earlier [5]. The pathophysiology of hypoglycemia is ambivalent. First, lowering blood glucose has a physiological function, signaling the need for food intake. Such functional hypoglycemia (FH), in other words, the feeling of hunger, is familiar to every healthy person, especially with irregular or insufficient nutrition, high physical exertion, and some other conditions and events. On the other hand, there is functional hunger hypoglycemia (FHH) as a pathological condition characterized by etiological and pathogenetic polymorphism and clinical diversity. FHH is especially typical for infants and children, which is explained by the insufficient maturity of the control mechanisms of homeostasis $[6,7]$.

In infants, children and adolescents, another form of $\mathrm{FH}$ is often observed, which occurs immediately or shortly after a meal. This functional reactive hypoglycemia (FRH), usually mild and transient, is also associated with constitutional, occasionally iatrogenic disorders of the mechanisms of regulation of energy metabolism $[6,8]$.

It must be admitted that although $\mathrm{FH}$ has quite distinct symptoms, the blood glucose level usually only approaches the lower limit of the norm, or slightly below the norm. Here it is necessary to clarify that true, or "chemical" hypoglycemia is called a decrease in the level of glucose in whole blood, plasma or serum by at least $0.3 \mathrm{mmol} / \mathrm{L}(5 \mathrm{mg} \%)$ from the lower limit of the norm adopted in this laboratory [5, 9]. Depending on the medium and the method of determination, this is $2.5-2.8 \mathrm{mmol} / \mathrm{L}$. But for newborns, hypoglycemia is considered a blood glucose level of $2.2 \mathrm{mmol} / \mathrm{L}$ and below [9, $10]$.

The most common etiological and pathogenetic variants of HS in infants, children and adolescents are shown in the classification.
Hypoglycemia in infancy, childhood and adolescence *

I. Impaired regulation of energy metabolism and transient hyperinsulinism

1. Vegetative-vascular dystonia (VVD), psychasthenia, other forms of autonomic dysfunction

2. Encephalopathy

3. Hypoglycemia in chromosomal aberrations (Down syndrome, Turner syndrome, etc.)

4. Hereditary genetic syndromes of children and adolescents (syndromes: Babinski-Fröhlich, BardetBiedl, Debré-Marie, Wilson's)

5. Pre-pubertal and pubertal obesity (false Fröhlich syndrome)

6. Kleine-Levin syndrome

7. Leucine-sensitive hypoglycemia

8. Beckwith-Wiedemann Syndrome (EMG-syndrome)

II. Ferment liable hypoglycemia**

1. Idiopathic ketogenic hypoglycemia of early and middle childhood

2. Reye's syndrome

3. Glycogenosis

4. Congenital fructose intolerance

5. Aminoacidopathia

6. Galactosemia or intolerance to galactose

7. Deficiency of endogenous alanine synthesis

8. Methylmalonic aciduria

9. Glycosylation disorder (CDG syndrome, Jacquin syndrome)

III. Non-ferment liable hypoglycemia

1. Hypoglycemia in infants:

a) newborn with erythroblastosis

b) newborns of mothers with diabetes

c) newborns of mothers with complicated pregnancy (gestosis, eclampsia)

d) premature or low birth weight

e) in severe or complicated labor (asphyxia, prenatal and postnatal hypoxia, hypothermia, infection, etc.)

2. Hypoglycemia with endocrinopathies:

a) hypothyroidism

b) hypoadrenocorticism

c) panhypopituitarism or selective hypopituitarism

3. Autoimmune hypoglycemia.

* not included iatrogenic and drug hypoglycemia, and organic hypoglycemia associated with beta-cell or ectopic hyperinsulinism in insulinoma or nesidioblastosis, in particular, in multiple endocrine neoplasia syndrome (MEN).

** ferment-liability are discussed in detail in a number of publications $[2-4,9,10,13,14]$.

\subsection{Pathophysiology of Hypoglycemia in Infants, Children and Adolescents}

The pathogenesis of HS, which often accompanies VSD, constitutional psycho-vegetative asthenia, and many orphan diseases and syndromes, is complex and not entirely understood. A multifaceted violation of the regulation of energy exchange is assumed. Here it should be taken into account that there is a hereditary genetic component in the 
etiology of these diseases and syndromes, and a genetic defect or mutation (usually partial) manifests itself in infancy, childhood or puberty in the form of a small, often transient defect in metabolic enzymes [2, 8, 11-14]. This defect is enhanced or realized against the background of age-related immaturity and functional inferiority of neuroendocrine metabolic regulation mechanisms [1, 5-7]. Sometimes the main role in the pathogenesis of HS is played by dysfunction of the hypothalamus, including hypertonicity nervus Vagus with hyperinsulinemia, as is the case with vegetative crises or panic attacks [1, 2, 5-7].

Complex "neuroendocrine" pathogenesis has HS in the chromosomal and hereditary-genetic syndromes indicated in the classification, juvenile mispituitarism, as well as in traumatic or vascular brain injuries, which are sometimes complicated by hypoglycemia. Along with the vagus-insular component of pathogenesis, there is often dysfunction or damage of the adenohypophysis, with a deficiency of CTT, ACTH, TSH, cortisol and thyroid hormones, which normally oppose to insulin [3, 6, 15-18].

Dysfunctions of the hypothalamus is responsible for HS in Kleine-Levin syndrome, which is manifested by hypersomnia, apathy, "narrowing" of consciousness, bulimia and aggressiveness. Frequent paroxysms of HS, in fact, chronic hypoglycemia, are associated with damage to the hypothalamus and increased tone of nervus Vagus [19].

Leucine-free hypoglycemia, which occurs in some children in response to food rich in leucine (egg white, soy, and some cheeses), has a mis regulatory nature. Hypoglycemia is caused by an increased secretory response of beta cells to leucine [10, $13,18]$.

Neonatal hypoglycemia in newborns with EMG syndrome is explained by the presence of nesidioblastosis in such infants $[2,4,19]$.

Numerous ferment infringements in the field of energy metabolism, often accompanied by hypoglycemia, are usually hereditary and genetic in nature $[3,4,13,14]$. These defects are usually congenital, or manifest in infancy and early childhood. Sometimes ferment-associated HS are self-limited with age. But more often the defect is lifelong, and hypoglycemia is chronic, although the decrease in blood glucose levels is usually small $[13,18]$.

Idiopathic ketogenic hypoglycemia occurs between the ages of 1 and 6 years and is classified as FGH. The syndrome is triggered by a low-carbohydrates diet or fasting, often in the presence of a feverish state. HS occurs in the morning: the child is lethargic, does not wake up, complains of a headache. Then vomiting, convulsions, loss of consciousness occurs. The baby smells with acetone. The causes of hypoglycemia are a defect in gluconeogenesis, utilization of free fatty acids and ketones $[2,4,9,12]$. The syndrome resolves spontaneously by the age of 6 [18].

Reye's syndrome is a disease of children and adolescents, which occurs with epileptoid encephalopathy, hepatomegaly, and fatty degeneration of internal organs. Hypoglycemia occurs with vomiting, impaired consciousness and breathing. There is a defect in gluconeogenesis and malabsorption due to liver and intestinal dystrophy $[4,10,13,14]$.

Hypoglycemia usually accompanies numerous variants of aminoacidopathy, for example, leucinosis, i.e. enzyme-dependent defect in the metabolism of leucine, isoleucine and valine. Peramination of these amino acids is the basis of gluconeogenesis, the violation of which is the cause of hypoglycemia. A characteristic sign of such fermentopathy is the smell of maple syrup in the urine of a child [10, 12, 19, 21, 22].

An even rarer enzyme deficiency hypoglycemia is methylmalonic aciduria due to methylmalone-CoA mutase deficiency. Moderate hypoglycemia is accompanied by massive urinary excretion of methylmalonic acid and acetone, which gives the urine a characteristic acetone odor $[2,4,11$, 14].

In addition to these ferment liability, HS can be caused by other extremely rare congenital disorders of carbohydrate and amino acid metabolism, such as intolerance to glycerol, deficiency of glucagon synthetase, propionic acidemia, tyrosinosis, etc. [2, 9, 10].

Non-ferment liable hypoglycemia in newborns with erythroblastosis is caused by the fact that hemolysis of erythrocytes leads to increased destruction of insulin. This provokes hyperfunction and then hypertrophy of beta cells with hyperinsulinism and regular hypoglycemia [4, 20-22].

Hypoglycemia in newborns whose mothers have diabetes mellitus is associated with chronic hyperglycemia in the mother and the fetus, which leads to hypertrophy of beta cells in the fetus [3, 9, 22-24]. Hypoglycemia in newborns can occur if the pregnant woman has taken salicylates, sulfonylureas or other drugs that stimulate insulin secretion and can cross the placental barrier [25].

Hypoglycemic paroxysms in premature infants, as well as in infants with a lack of weight, or those born after a complicated and multiple pregnancy, after severe or complicated childbirth, are explained by the rapid depletion and immaturity of hormonal and enzyme systems that regulate energy metabolism [9, 12, 22, 26, 27].

The pathogenesis of hypoglycemia in endocrine diseases specified in the classification is associated with a deficiency of certain counter insular hormones [2, 4, 16-18].

Autoimmune hypoglycemia or Hirata disease caused by antibodies to insulin, insulin receptors, and $\beta$-cell antigens is very rare. Antibodies arise spontaneously, blocking insulin with the development of diabetes mellitus (DM). Occasionally, the insulin-antibody complex disintegrates with the release of insulin and severe HS. Antibodies to insulin receptors and $\beta$-cell antigens can initially increase tissue sensitivity to insulin and stimulate its secretion. That is why, before the onset of type 1 diabetes, hypoglycemia is often noted [3, 5, 22, 26].

\subsection{Clinical Presentation and Diagnosis of Hypoglycemia}

The clinical manifestations of HS are nonspecific, highly variable and poorly correlated with the level of glycemia, the main biochemical criterion of the syndrome. Given the physiology of infancy and childhood, the classical division of 
symptoms of HS into adrenergic and neuroglycopenic is unproductive. An indication of HS in newborns and infants is lethargy, drowsiness, strabismus, anxiety and sudden attacks of screaming, tremors or convulsions, refusal to breast and vomiting, rare or, on the contrary, rapid breathing, pallor or cyanosis of the skin, sweating, tachycardia, insufficient weight gain and growth retardation. Some orphan syndromes prone to hypoglycemia are characterized by jaundice, edema or ascites, hemorrhagic diathesis, anthropometric stigmas microgenia, epicanthus, increased body weight, macroglossia and visceromegaly, umbilical cord cyst. In certain cases, there is a smell of acetone or maple syrup. Children and adolescents have headache, hunger, tremors, obesity, short stature, xanthomatotic, physical or mental retardation are often recorded [2, 3, 4, 8, 14, 18, 19, 21, 26, 27, 28]. In laboratory tests, along with hypoglycemia, hyperketonemia, hyperpyruvatemia and hyperlacticaemia, increased levels of insulin (IRI), C-peptide, alanine and transaminases, decreased levels of B vitamins, glucosuria, acetonuria, aminoaciduria and methylmalonic aciduria are recorded [4, 9, 10, 22, 29].

The HS is verified by two to threefold determination of glucose in the blood on an empty stomach; in adolescents, it is permissible to conduct a glucose tolerance test and diagnostic tests. Pathogenetic diagnostics, as a rule, is complex, requires many biochemical, hormonal, enzymological, immunochemical, genetic, introscope and functional methods, tests and samples [22, 28]. The scheme of etiological and pathogenetic diagnosis of HS is shown below (Scheme 1).

\subsection{Treatment and Prevention of Hypoglycemia}

HS often takes on an acute form in the form of $\mathrm{HC}$, which requires immediate assistance. Urgent therapy of $\mathrm{HC}$ is given in the table 1 .

Table 1. Urgent therapy for hypoglycemic coma [2, 4, 9, 10, 18, 29-31].

\begin{tabular}{|c|c|c|}
\hline Primary therapy & Syndromic therapy & Diagnostics \\
\hline $\begin{array}{l}\text { The first } 5-10 \text { minutes } \\
\text { 1. Intravenous } 10 \% \text { glucose solution at the rate of } 1 \mathrm{ml} / \mathrm{kg} \text { body weight } \\
\text { 2. If venepuncture is impossible, intramuscular injection or subcutaneous } \\
\text { injection of } 0,25-0,5 \mathrm{mg} \text { glucagon (children up to } 5 \text { years) } 0.5-1 \mathrm{mg} \\
\text { glucagon children over } 5 \text { years, or subcutaneously } 0.5 \mathrm{ml} \text { of a } 1 \% \text { solution } \\
\text { of adrenaline * }\end{array}$ & $\begin{array}{l}\text { 1. In case of loss of consciousness and } \\
\text { seizures, take measures against } \\
\text { aspiration and trauma } \\
\text { 2. Hospitalization in intensive treatment } \\
\text { unit }\end{array}$ & $\begin{array}{l}\text { 1. Determine the level of glucose } \\
\text { in the blood by rapid method } \\
2 \text {. Hemodynamic control }\end{array}$ \\
\hline $\begin{array}{l}\text { The following } 10-30 \text { minutes } \\
\text { 1. If consciousness is not restored -intravenously infusion of } 10 \% \text { glucose } \\
\text { solution at a rate } 3 \mathrm{ml} / \mathrm{kg} / \text { weight / hour } \\
\text { 2. Glycemia }>5 \mathrm{mmol} / \mathrm{L} \text {, but conscious } \\
\text { did not recover. If there are signs } \\
\text { cerebral oedema: } \\
\text { 1. intravenously, dropwise mannitol } 0.5-1 \mathrm{~g} / \mathrm{kg} / \text { weight in } 10 \% \text { glucose } \\
\text { solution; } \\
\text { 2. into the stomach through a tube glycerol } 0.5-1.5 \mathrm{~g} / \mathrm{kg} \text {; } \\
\text { 3. intravenous furosemide } 2 \mathrm{mg} / \mathrm{kg} \\
\text { 4. hydrocortisone } 20 \mathrm{mg} / \mathrm{kg} \\
\text { or dexamethasone } 0.5-2 \mathrm{mg} / \mathrm{kg} \\
\text { or prednisolone } 5 \mathrm{mg} / \mathrm{kg} \\
\text { intravenously, dropwise albumin } 10-20 \% \text { solution } 5-10 \mathrm{ml} / \mathrm{kg} / \text { day }\end{array}$ & $\begin{array}{l}\text { 1. Convulsions persistent: } \\
\text { Diazepam } 0.05-0.1 \mathrm{~kg} / \text { weight with } \\
10 \% \text { solution of glucose intravenously } \\
\text { or intramuscularly. In the absence of } \\
\text { effect - anaesthesia } \\
2 \text {. With hyperthermia } \\
\text { intravenously, } 20 \% \text { GOB } 0.25-0.35 \mathrm{ml} / \\
\mathrm{kg} \\
\text { 3. Oxygen therapy } \\
\text { 4. According to indications hypotensive, } \\
\text { analeptics, cardiotonic, etc. }\end{array}$ & $\begin{array}{l}\text { 1. ECG } \\
\text { 2. CT, neurologist and } \\
\text { ophthalmologist consultation to } \\
\text { exclude cerebral oedema, or } \\
\text { haemorrhage } \\
\text { 3. If cerebral edema or intracranial } \\
\text { hematoma, shock and distress } \\
\text { syndrome are excluded, and } \\
\text { glycemia is }>5 \text { mmol / L, reactive } \\
\text { psychosis or hysteria should be } \\
\text { excluded. Examination by a } \\
\text { psychiatrist. }\end{array}$ \\
\hline
\end{tabular}

* with glycogenosis and other HS with glycogen deficiency, glucagon and adrenaline are ineffective

Further treatment depends on the pathogenetic diagnosis, but in most cases, treatment is symptomatic.

Diet therapy is the main method of prevention and treatment of mis regulatory, i.e. functional hypoglycemia, and practically the only method for the treatment of enzymopathy hypoglycemia, idiopathic hypoglycemia and nesidioblastosis. With FGH, a diet balanced in terms of energy is prescribed and strict adherence to the food intake regime.

In the treatment of FRH a diet is also important, the main conditions of which are the regime of frequent meals (5-6 times a day) and restriction of calorie intake (no more than 25 $\mathrm{kcal} / \mathrm{kg}$ of body weight) with equal calorie portions. They reduce the content of carbohydrates ( $100 \mathrm{~g}$ per day), exclude refined carbohydrates, add carriers of coarse fiber (guar, pectin, methylcellulose).

In specific ferment liability, products containing fructose, galactose, leucine, etc. are limited or excluded.

The risk group for postnatal hypoglycemia of newborns includes infants of mothers with diabetes mellitus, mothers with preeclampsia, eclampsia, or who received frequent and massive glucose infusion during pregnancy. This group includes premature infants, newborns with signs of COFS syndrome, stigmas of other orphan syndromes listed in the classification, as well as a smaller twin. According to the WHO recommendation, such newborns should determine glycemia no later than 1 hour after childbirth, followed by monitoring every 3 hours. They are provided with contact with the mother immediately after childbirth, breastfeeding from the first hour of life, and then feeding every 2-2.5 hours without a night break. Since breastfeeding in the first hours is problematic, it is recommended to give the baby $5 \%$ glucose solution orally at the rate of $3-5 \mathrm{ml} / \mathrm{kg}$ body weight one hour after birth. McCann et al. [31] in order to prevent postnatal hypoglycemia suggested to inject an isotonic solution of fructose into the umbilical vein in the first 15 minutes after birth, at the rate of $2 \mathrm{~g}$ per $\mathrm{kg}$ of weight. 


\section{Discussion}

From the point of view of physiology and pathophysiology, hypoglycemia and HS have a dual character. On the one hand, the approach of the blood glucose level to the physiological minimum serves as a signal for food intake and, at the same time, as a trigger factor for the mobilization of stored energy substrates - glycogen and adipose tissue. On the other hand, even not very deep, but prolonged, especially chronic hypoglycemia causes or intensifies numerous functional, biochemical and anatomical and morphological changes and disorders - vegetative dystonia, neurasthenia, hyperketonemia and ketoacidosis, hyperuricemia, hepatitis, in the form of fatty liver infiltration, hepatomegaly and increasing liver enzymes $[3-5,11,20]$. It is precisely the age groups considered in this article that are especially susceptible to adverse long-term consequences of chronic hypoglycemia, in the form of a delay in intellectual and physical development, and various forms of deviant behavior $[1,2,8]$. At the same time, HS in infants, children and adolescents very often proceeds according to an urgent scenario, when a significant or very rapid decrease in the concentration of glucose in the blood is accompanied by vivid psychovegetoneurotic symptoms, and then loss of consciousness, i.e. the development of the HC.

Thus, the prevention, timely recognition and effective treatment of HS in children are still relevant. Unfortunately, the clinical picture of HS in both adults and children is variable, non-pathognomonic and correlates poorly with the blood glucose level, especially since HS is often self-limited during development. It should be recognized that the accepted separation of neuroglycopenic and adrenergic symptoms as stages in the development of HS is not quite correct, since they actually occur simultaneously $[5,19,29]$. This is especially true for infants, children, and adolescents, in whom hypoglycemia often manifests as psychopathological, deviant phantoms $[4,8,20]$.

This complicates the diagnosis of HS, so it is important to know the most common syndromes and diseases of infants, children and adolescents, fraught with HS. A list of such diseases and syndromes is given in the HS classification.

Since causal therapy is not possible for most of the etiological and pathogenetic variants of HS recorded in the classification, it is necessary to limit ourselves to symptomatic treatment and diet therapy. This fully applies to the most common form of the syndrome - functional hypoglycemia $[2-4,8]$. It is pertinent to note here that for the prevention and treatment of $\mathrm{FHH}$, it is very important to provide free breakfasts to primary school students, which is adopted in many countries.

\section{Conclusion}

In conclusion, it should be emphasized that the main factor in the pathogenesis of HS in infants and children is immaturity and functional inferiority of adaptive reactions and mechanisms. It should also be borne in mind that prolonged hypoglycemia, especially $\mathrm{HC}$, is fraught with severe complications - chronic or acute metabolic disorders and cerebral blood flow, cerebral edema, pituitary apoplexy, dangerous cardio arrhythmia or stem disorders, sometimes with the death of the patient. Hypoglycemia should be considered as a threat to growth, development and even to the life of the child, therefore, timely diagnosis is necessary, and with $\mathrm{HC}$, qualified emergency care.

In general, the management of hypoglycemia and the prevention of $\mathrm{HC}$ requires a multidisciplinary and personalized approach.

Complaints and symptoms suggestive of hypoglycemia: newborns in difficult labor, mothers with diabetes mellitus, low birth weight, lethargy, drowsiness, nystagmus, strabismus, tremors or convulsions, breast refusal and vomiting, infrequent or frequent breathing, pallor, hyperemia or cyanosis of the skin, insufficient weight gain or stunted growth, jaundice, edema or ascites, hemorrhagic or allergic diathesis, macroglossia, visceromegaly, umbilical cord cyst, the smell of acetone or maple syrup. In children or adolescents: headache, tremors, obesity, stretch marks, retardation in physical and sexual development, xanthomatosis, signs of orphan, chromosomal or endocrine diseases, delayed physical and mental development or mental retardation

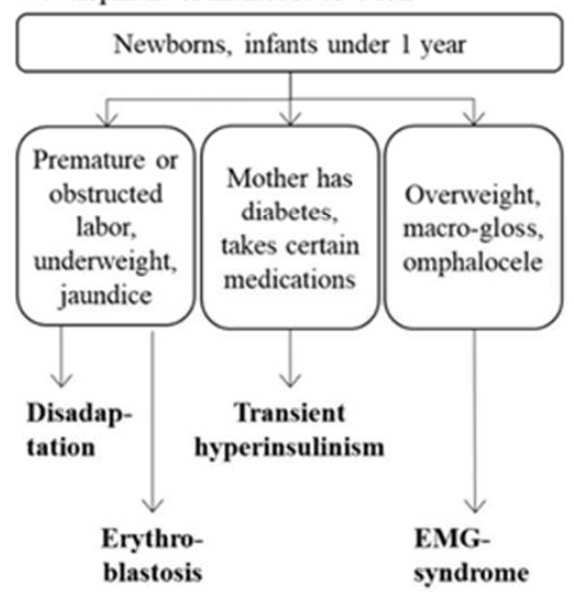

blastosis

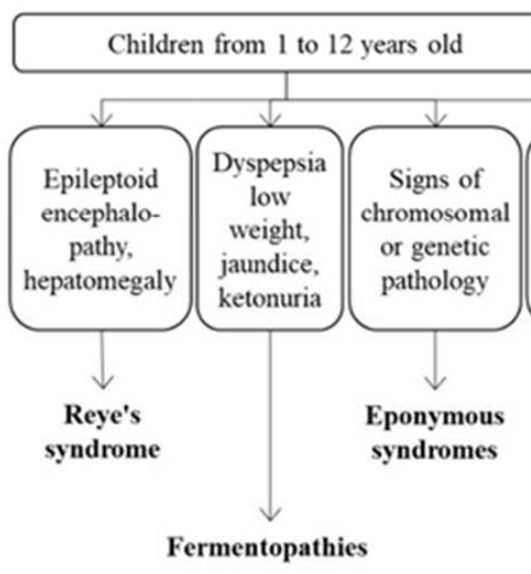

Figure 1. Hypoglycemia Diagnostic Scheme. 


\section{Declaration}

The authors declare no financial support and no conflicts of interest.

\section{References}

[1] Chutko L. S., Surushina S. U., Anisimova T. I. Vegetativno-vascular dystonia in children and adolescents. Clinical and pathophysiological signs and therapy (Revew). // Practica pediatra. 2019. -N3. -pp. 17-21. (in Rus). https:/medi.ru/pp/201904/16408/

[2] Marks V., Rose F. Hypoglycemia. 2-nd Ed. Blackwell sci. publ N-Y: - 1981. ISBN 978-0632006731.

[3] Shuvin Z., Felig F. Hypoglycemia. In: Endocrinology and Metabolism (transl. from Eng.). Eds. F. Felig et al. M.: Medicine. - 1985. - vol. 2. - pp. 218-258. (in Rus). https:/uchi-fitness.ru/about/articls/endocrinologia-i-metabolis $\mathrm{m} /$

[4] Dedov I. I., Petercova V. A. Manual for pediatric Endocrinology. M.: Universum publishing. -2001. (in Rus). ISBN 5-7736-0056-0.

[5] Lukyanchikov V. S., Contemorary Definitions of Hypoglycemia as frequent and dangerous Syndrome and Coma//Amer. J. Life Science. -2019. 7 (5). 83-90. doi: 10.11648/j.ajls.20190705.11.

[6] Sisley S., Sandoval D. Hypothalamic Control of Energy and Glucose Metabolism. // Rew. Endocr. Metabol. Disord. -2011. -12 (3). -pp. 219-233. doi: 10.1007/s11154-011-9189-x.

[7] Jin S., Diano S. Mitochondrial Dynamics and Hypothalamic Regulation of Metabolism // Endocrinology. -2018. -vol. 159 (10). -pp. 3596-3604. doi: 10.1210/eh.201800667.

[8] Brund F., Fedou C., Mercier J. Postprandial reactive Hypoglycemia. // Diabetes and Metabolism. -2000. №. 26. -P. 337-351. http:/www.a stadium. org (media)/

[9] Kamyshnikov V. S. Clinical laboratory tests from A to Z and their diagnostic profiles./ M.: - Medpress-inform. - 2018. (in Rus). ISBN 978-5-00030-559-1.

[10] Taranushenko T. E., Kiselyova N. G., Lasareva O. V., Kaljugnaya I. I. Hypoglycemia in newborn: literature review and description of clinical case // Problemi Endocrinologyi. -2019. - 65 (4). -pp. 251-262. (in Rus). doi: $10.14341 / \mathrm{prob} / 8336$

[11] Thornton P. S., Stanley CA., De Leon D. D. Recommendations from Endocrine Society for Evaluation and Management of persistent Hypoglycemia in Children // J. Pediatr. -2015. -vol. 167.pp. 238-245. http://www:ncbi.nim.nih.gov./pmc/articels/PMC4659381.

[12] Glycogen Disease. Clinical Recommendations. M.: Unite of pediatricians of Russia. -2016 (in Rus). There is no index

[13] Zacharova E. U., Krasilnikova E. U. Orphan Diseases.// Practica pediatra -2019. -N4. (in Rus). doi: eLabrary/D:41443118.

[14] James C., Kapoor R. R. Ismail D. The genetic Basis of congenital hyperinsulinism. // J. Med. Genet. -2009. -№ 46. -pp. 289-299. doi: 10.11.36/img.2008.064337.
[15] Geffner M. E. Panhypopituitarism in Children. // Cancer Control. -2002.-vol. 9. -pp. 212-222. There is no index.

[16] White P. S., Speiser R. W. Congenital Adrenal hyperplasia due to 21-hydroxylase deficiency. // Endocr. Rev. -2000. - № 21. -pp. 245-291. doi: 101177/107327480200900304.

[17] Bornstein S., Allolio B., Arlt W., Wiebke A. Diagnosis, Treatment and follow-up of Patients with primary Adrenal insufficiency. // J. Intern. Med. -2015. -vol. 275. -pp. 104-115. doi: $101210 /$ jc2015-1710.

[18] B. Leiber, G. Olbrich. Die klinischen syndrome. M.: Medicina. 1974] (in Rus). There is no index.

[19] Foster D., Rubinstein A. Hypoglycemia, Insulinoma and other hormone-producing tumors of pancreas. In "Internal Diseases". Eds. E. Braunvald et al. M.: -Medicina. -1997. Ch. 9. pp. 232-247. (in Rus). ISBN 0-07-100134-4.

[20] Wolfsdorf J. I., Weinstein D. A. Hypoglycemia in Children//In "Pediatric Endocrinology. Ed. F. Lifshiz. 5-th ed. NY. Mar. Dek. -2007. -vol. 1. -pp. 329-333. ISBN-13 (EAN): 9781420042719 .

[21] Diagnosis and Treatment of endocrine Diseases of Children. 3-d Ed. By N. P. Schabalov. M.: Medpress-Inform. -2017. (in Rus) ISBN 9-978-00030-392-4.

[22] Samoilova U. G., Sivolobova T. V., Kudlay D. A. Violation of carboanhydrate metabolism in newborn with low and extremally low weight // Practica Pediatra 2020. Vol. 99. N1. pp. 194-198. (in Rus). https: /medi.ru/pp/202002/16408/.

[23] Martinova M. I., Smirnov V. V., Kartelishev A. L. Diagnostic Errors and clinical and biochemical Features of emergency Conditions in Children with Diabetes.// Pediatria. -2005. -№ 6. - pp. 103-105. (in Rus). http: eLIBRARY ID: 9173306

[24] Federal clinical recommendations for Management of the Children and Adolescents with Hyperinsulinism. // Problemy Endocrinol. -2014. -60 (2). -pp. 31-41. (in Rus). doi: 10.1434/probl2014; 60 (2): 31-41.

[25] Ostroumova O. D., Akimova E. S., Kochetkov A. I., Pereversev A. P. Drug-induced Hypoglycemia: focus on non-sugar-lowering drugs // CONSILIUM medicum. 2019. Vol. 21. - №4. -pp. 59-65. (in Rus). doi: 10.26442/20751753.2019.4.190196.

[26] Boyle P. J. Hypoglycemia. In: Medical Management of Diabetes Mellitus. Eds. J. Leaty etc. Marcel Dekker Inc. NY, Basel. 2000. Ch. 31. -pp. 527-537. Available at: http://wwwdekker.com.

[27] Filan P. M., Inder T. E., Cameron F. G. Neonatal Hypoglycemia and occipital Cerebral Injury // J. Pediatrics.-2006. -№ 148. -pp. 552-555. doi: 103174/ajnr A3423.

[28] Shustov S. B., Halimov U. Sh., Saluhov V. V., Trufanov G. E. Functional and Topical Diagnostic in Endocrinology. M.: GEOTAR Media. 2017. (in Rus.). ISBN 978-5-97044118-3.

[29] Inzucchi S., Kinder B. Endocrine Emergencies: Hypoglycemic and Hyperglycemic Crises. In: Textbook of Endocrine Surgery. (Eds. O. Clark, Q-Y. Duh.). / WE Sounders Company. Washington etc.: - 1997. - pp. 175-181. ISBN-10: 9351528065. 
[30] Intensive Therapy. Contemporary Aspects. By D. Sew, D. Wing (Eds.). M.: Medpress - Inform. 2008. (in Rus.). ISBN 5-98322-295-3.
[31] McCann M. L., Adam P. A., Likely B. Prevention of Hypoglycemia in Infants of diabetic Mothers. // New Engl. J. Medic. 1966. -vol. 275. -№ 1. -pp. 8-12. http:/Printiss N: 0028-4793. 